\title{
Traceable profile and roughness measurements inside micro sonic nozzles with the Profilscanner
}

\author{
Min Xu ${ }^{a 1}$, Felix Kauth ${ }^{2}$, Bodo Mickan ${ }^{1}$ and Uwe Brand ${ }^{1}$ \\ ${ }^{1}$ Physikalisch-Technische Bundesanstalt (PTB), Bundesallee 100, D-38116 Braunschweig, Germany \\ ${ }^{2}$ Leibniz Universität Hannover, Appelstraße 9, D-30167 Hannover, Germany
}

Résumé. Le Profilscanner développé par le PTB permet de réaliser des mesures de rugosité et de de forme sur des structures d'un diamètre minimum de $40 \mu \mathrm{m}$ présentant un rapport d'aspect élevé. Il a été possible pour la première de caractériser avec succès les surfaces internes de petites buses soniques d'un diamètre de l'ordre du micromètre. La comparaison entre ces mesures topographiques et des expériences correspondantes concernant le débit massique a confirmé l'hypothèse selon laquelle la topographie des surfaces internes a une forte influence sur le débit massique des microbuses.

\section{Introduction}

High-aspect-ratio microstructures (HARMS), such as micro holes, vertical micro-mirrors, or micro-concave products, are increasingly utilized in practice in the fields of biotechnology, aerospace and automotive industries. However, the non-destructive quality control of such structures still remains a challenge because the extremely narrow probing spaces are difficult for the existing tactile and optical measurement instruments. In the past two decades, some novel measurement technologies have been developed focusing on the measurement of HARMS. The VibroScanning (VS) method [1] is one of the early solutions and obtains the surface profile through detecting the electrical contact between a vibrating probe and the inner surface of a hole and measuring the duty factor of the contact. Later a twin-probe system [2, 3] was further developed as an update to make it possible to characterize the artefact regardless of materials. However, the precision of this system is limited to about $0.5 \mu \mathrm{m}$. The fibre probe based technology [4-11] is another approach for the measurement of HARMS and some of these measurement systems have already been utilized commercially. In this technology a slim fibre with a micro ball at the end is employed for probing, and the surface positions are inferred through detecting the positions of the ball probe by various sensing methods, e.g., imaging the shadow of the probing systems using a camera. In some of these measurement systems the micro ball and the shank are oscillated to avoid the stick-slip effect. However, this technique is mainly applied in geometry detections such as the roundness. The micro ball probes with the radii mostly above $15 \mu \mathrm{m}$ make it not suitable to measure fine roughness. Industrial computed tomography (CT) [12] has been also sometimes applied for HARMS detections but its measurement lateral resolution is not sufficient for roughness measurements.

For non-destructive and accurate roughness and profile measurements of HARMS structures, a traceable
Profilscanner has been developed at the PhysikalischTechnische Bundesanstalt (PTB) [13], and the inner surfaces of sonic nozzles with diameters in the micrometre range have been successfully characterized using Profilscanner.

Sonic nozzles [14] are widely employed as calibration standards for gas and fluid flow measurements due to their long term accuracy and excellent repeatability. With the decrease of the nozzle size, the influence of the topography of the inner surface on the flow rate increases. Therefore the knowledge about the inner surface is especially important for sonic nozzles with diameters in the micrometre range. Here for the first time profile and roughness measurements inside micro nozzles using the Profilscanner will be presented.

In what follows, the system design and construction of the Profilscanner are described, and the surface profile and roughness measurements inside sonic nozzles are presented.

\footnotetext{
a Corresponding author: min.xu@ptb.de
} 


\section{Instrument construction}

The Profilscanner consists of four main modules: (1) a three dimensional (3D) piezo motion stage with the controllers providing precise positioning ability; (2) a piezoresistive microprobe with signal processing units for surface probing; (3) a 3D coarse motion stage with the controllers for coarse positioning of the artefacts, and (4) a laser interferometer measurement system allowing traceable measurements. Figure 1 shows the construction of the Profilscanner.

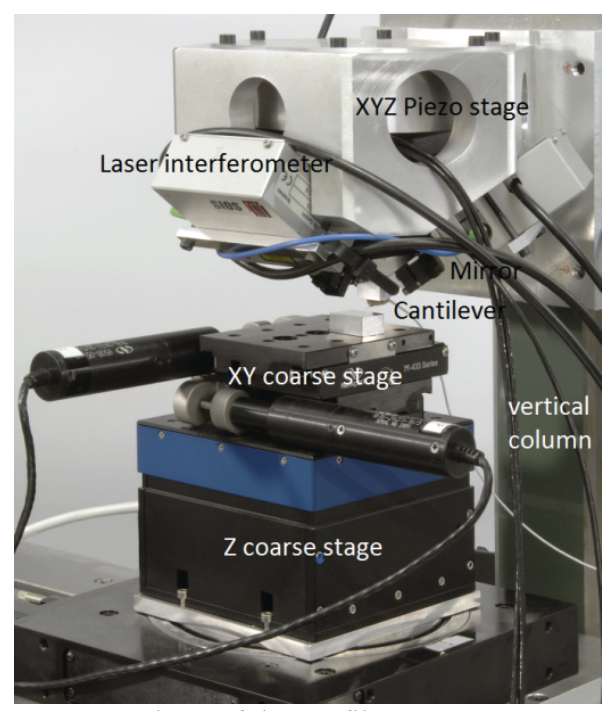

Figure 1. Photo of the Profilscanner setup

The head of the instrument contains a XY piezo stage (PI, model: P-628.2CD) and a Z piezo stage (PI, model: P-622.ZCD), which are controlled by the digital piezo controllers PI E-710 and PI E-753 separately. The travel range of the $3 \mathrm{D}$ stage is $800 \mu \mathrm{m} \times 800 \mu \mathrm{m} \times 250 \mu \mathrm{m}(\mathrm{x} \times$ $\mathrm{y} \times \mathrm{z})$. The advantage of using a scanning head instrument is that this implies no limit on the artefacts mass. Usually the artefact mass would have a negative effect on the motion stages dynamic properties. The entire head of the system can be moved about $100 \mathrm{~mm}$ in $\mathrm{z}$-direction along the vertical column to measure large artefacts up to $80 \mathrm{~mm} \times 100 \mathrm{~mm} \times 100 \mathrm{~mm}$. On the base of the instrument the motorized coarse positioning stage with a movement range of $50 \mathrm{~mm} \times 50 \mathrm{~mm} \times 12 \mathrm{~mm}(\mathrm{x} \times$ $\mathrm{y} \times \mathrm{z}$ ) is mounted.

The key element of the Profilscanner is a long silicon cantilever type microprobe [15-19] (called as cantilever in the following) with an integrated probing tip at the free end, which is designed by PTB together with the Forschungsinstitut für Mikrosensorik und Photovoltaik $\mathrm{GmbH}$ (CiS) Erfurt and the Institute for Semiconductor Technology of Technical University of Braunschweig. A full-bridge piezoresistive strain gauge on the cantilever close to its clamping measures the bending of the cantilever. With a constant voltage supply to the balanced Wheatstone bridge, a measurement voltage change can be observed when a force acts vertically on the probing tip. Three different cantilever lengths have been fabricated for the measurement of different size of HARMS. The cantilevers are $1.5 \mathrm{~mm}, 3 \mathrm{~mm}$ and $5 \mathrm{~mm}$ long, $30 \mu \mathrm{m}$,
$100 \mu \mathrm{m}$ and $200 \mu \mathrm{m}$ wide, and $25 \mu \mathrm{m}, 25 \mu \mathrm{m}$ and $50 \mu \mathrm{m}$ thick. The tips were fabricated with heights of $22 \mu \mathrm{m}, 70$ $\mu \mathrm{m}$ and $116 \mu \mathrm{m}$. Th is kind of high tips permits it to characterize deep structures such as concave surfaces or vertical micro-mirrors. Thus the slender cantilever enables the Profilscanner to perform measurements at the narrow sidewall of HARMS with diameters down to 40 $\mu \mathrm{m}$. Figure 2 shows a sample of a $1.5 \mathrm{~mm}$ long cantilever. With tip radii of less than $100 \mathrm{~nm}$, the cantilever provides high lateral resolution for surface profile and roughness measurements. Besides the above advantages, the cantilever is realized using silicon bulk micromachining, which ensures low cost and high production quality.

The cantilever is bonded on the cantilever holder and mounted on the XYZ piezo stages. When the instrument scans, the cantilever moves with the piezo stages while the artefact surface keeps stationary.

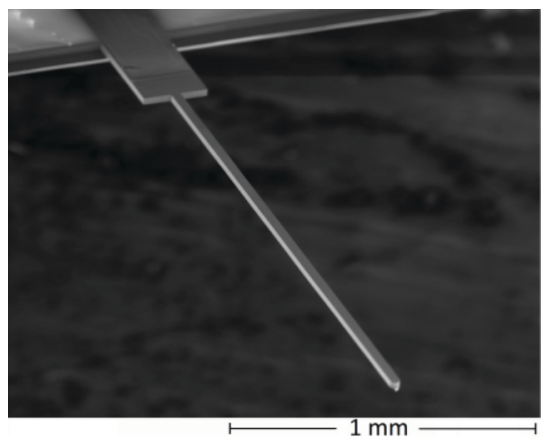

(a)

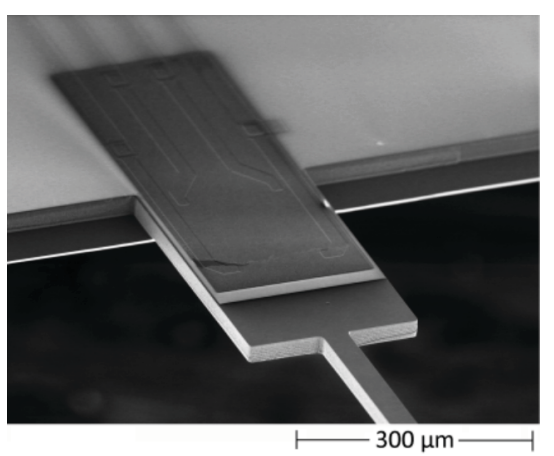

(b)

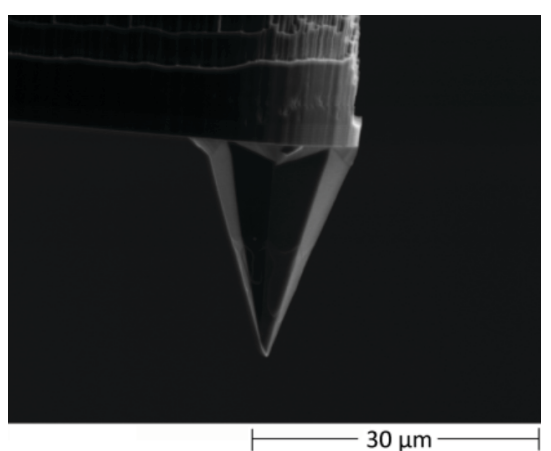

(c)

Figure 2. (a) Sample of $1.5 \mathrm{~mm}$ long tactile piezoresistive microprobe; (b) strain gauge near the clamping; (c) integrated probing tip. 
Before the application of a cantilever, the sensitivity of the cantilever has to be characterized by incremental movements of the $\mathrm{Z}$ piezo stage up to $10 \mu \mathrm{m}$ in steps of $0.1 \mu \mathrm{m}$ with the cantilever probing a smooth artefact surface. Simultaneously the cantilever output voltage is detected by an A/D converter (National Instruments, model: PCI-6281). Then the cantilever sensitivity is applied to adjust the parameters of the $\mathrm{Z}$ piezo stage controller.

The Profilscanner is built as a closed-loop system. The cantilever out-of-balance voltage signal is filte red and amplified by a low noise amplifier (HBM, model: MGCplus), and fed to the $\mathrm{Z}$ piezo controller to serve as the control signal of the $Z$ piezo stage. During measurement procedure, the $Z$ piezo stage and the cantilever move up and down with the artefact surface and a constant contact force is kept between cantilever and the artefact surface. The probing force $F_{c}$ is determined by the out-of-balance voltage signal $r$, the cantilever stiffness $k$ and cantilever sensitivity $s$. For a cantilever with stiffness $k=8.5 \mathrm{~N} / \mathrm{m}$, a sensitivity $s=250$ $\mathrm{V} / \mathrm{m}$ and an out-of-balance voltage signal $r$ of $0.2 \mathrm{mV}$ during the whole measurement, a contact force

of $6.8 \mu N$ is resulted.

$$
F_{c}=r \times k / s
$$

The probing force of the Profilscanner can be set lower than $1 \mu \mathrm{N}$. Such a small probing force prevents the tip from scratching the artefact surface.

On the head of the system, three laser interferometers with $1 \mathrm{~nm}$ resolution (SIOS, model: SP2000) are arranged perpendicular to each other to provide metrological traceability. To achieve an Abbe error-free measurement, the three laser beams intersect virtually on the cantilever tip. During the measurements, the cantilever is moved by the XYZ piezo motion stages, and the three laser interferometers measure the position of the cantilever. The topography data of the artefact are constructed from the three laser interferometer readouts. To ensure that the readings of the three laser interferometers and the cantilever signal are exactly from the same position, the samplings of the four signals should occur at the same time. To realize this, a wave generator is used to generate a trigger signal to synchronize the four readouts.

\section{Measurements inside micro sonic nozzles}

Sonic nozzle, also named Critical Flow Nozzle, Critical Flow Venturi or Sonic Venturi (see figure 3(a)), has been used in a number of industrial and scientific applications for gas and fluid flow measurements, e.g., as a calibration standard in gas meter calibration or as a transfer flow meter. The inner structure of the sonic nozzle defined in ISO 9300 consists of a smooth rounded inlet converging to a minimum throat area and then along a diverging exit cone. The diameter at the minimum throat, $\mathrm{d}$ in figure $3(\mathrm{~b})$, is called the throat diameter of the nozzle. The opening angle of the exit cone, $\theta$ in figure $3(b)$, is the diffuser opening angle.
At a given upstream pressure and temperature, an accelerated flow passes through the nozzle, with increasing velocity and decreasing density, into a lower pressure environment. The maximum velocity is achieved at the throat, the minimum area, where it reaches the desired speed of sound, and at the same time the Venturi effect causes the "choked" flow. The outlet mass flow rate will not increase with a further decrease in the downstream pressure environment while upstream pressure is fixed. In this state, only the upstream pressure and temperature are influencing the mass flow rate through the nozzle. The mass flow rate through the nozzle becomes nearly a linear function of the upstream pressure. Therefore a desired mass flow rate can be produced under choked conditions. Figure 4 shows the theoretical mass flow curve of a sonic nozzle. The vertical axis is normalized by the maximum mass flow rate. At the beginning of the experiment (position $\mathrm{A}$ ), the

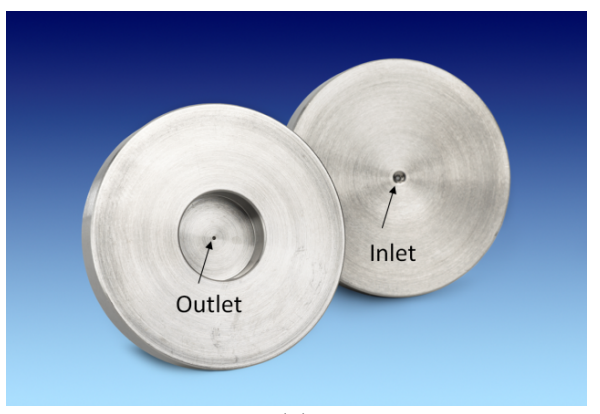

(a)

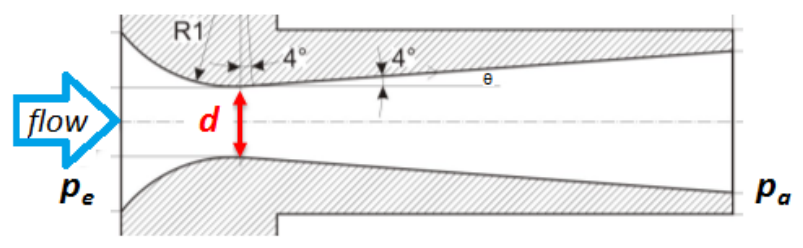

(b)

Figure 3. ISO 9300 type sonic nozzles, (a) external shape of the nozzle; (b) sketch showing a cross section through a nozzle.

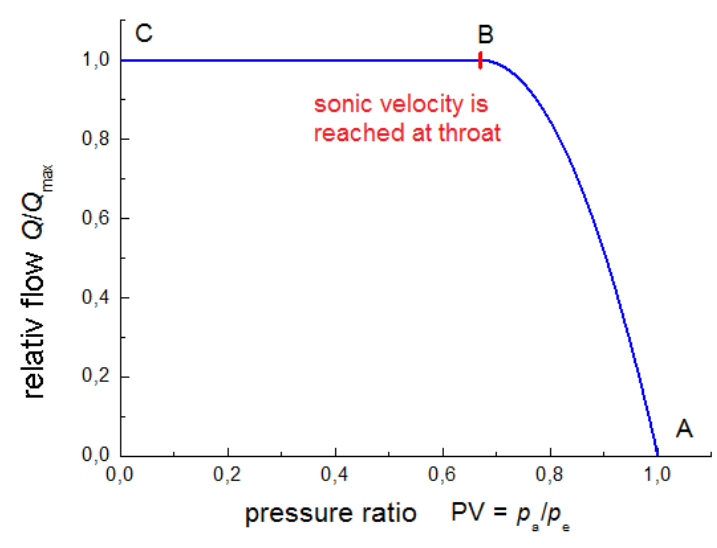

Figure 4. Theoretical flow curve of a sonic nozzle 
downstream pressure $p_{a}$ equals to the upstream pressure $p_{e}$, and no flow runs through the nozzle. With the increment of the pressure ratio $\mathrm{PV}=p_{a} / p_{e}$ (the downstream pressure decreases at a given upstream pressure), the mass flow rate increases till sonic velocity is reached at the throat area. Then the critical pressure ratio is reached at position $B$. Thereafter the flow rate keeps constant although the downstream pressure decreases further. At position $\mathrm{C}$ the downstream pressure $p_{a}$ decreases to 0 mbar.

Sonic nozzles with diameters in the micrometer range are increasingly employed in different areas such as domestic gas meters. The impact of the inner surface on the flow rate grows with the decrease of the nozzle size. In mass flow rate experiments of micro nozzles, deviations from a stable flow rate are sometimes observed. The mass flow rate results of two small nozzles are shown in figure 5. The vertical axis is the relative deviation from the theoretical mass flow rate. The mass flow rate curve of nozzle 1 is close to the theoretical values. In the mass flow rate curve of nozzle 2, however, a sharp flow rate drop occurs at a pressure ratio lower than the critical pressure ratio, where the mass flow rate should keep stable theoretically. It is called "low unchoking"[20], which forces to use quite low downstream pressure in the mass flow rate calibrations and leads to high demands on the vacuum equipments and high energy consumptions. For this reason it is necessary to characterize the inner surface and profile of the micro nozzles and investigate the influence of the inner surface profile on the flow rate behaviour.

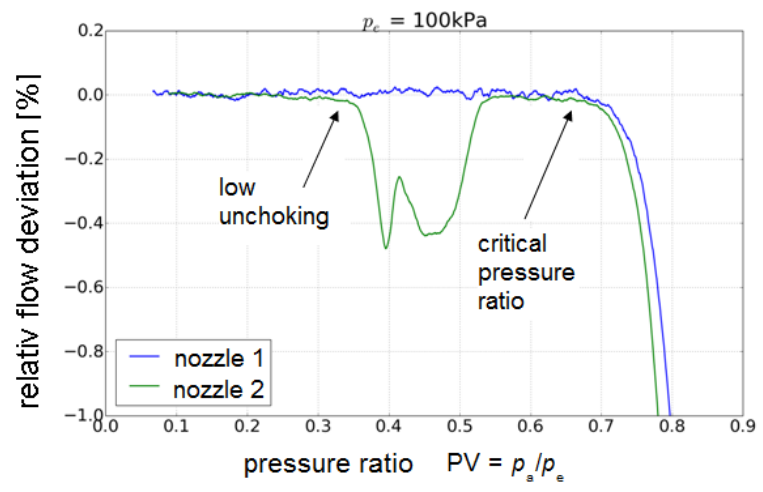

Figure 5. Mass flow rate curves of two small nozzles

Nozzles manufactured by two methods, turning and Electrical Discharge Machining (EDM), were measured using the Profilscanner. The surface textures manufactured by the two methods are different. The inner surface of nozzles fabricated by turning is smoother than that fabricated by EDM. The surface measurement results of the Profilscanner indicate that the arithmetical mean deviation $R_{a}$ of the EDM-fabricate nozzle inner surface is about $0.62 \mu \mathrm{m}$, while that of the turning-fabricate nozzle inner surface is only about $0.18 \mu \mathrm{m}$.

The throat diameters of the measured nozzles vary from $200 \mu \mathrm{m}$ to $800 \mu \mathrm{m}$. Because the interested area in the measurement is from the throat to the end of the exit cone, the measurement lengths are at least 7 times of the throat diameter. A $3 \mathrm{~mm}$ long cantilever is used for the measurements of the nozzles with throat diameters of 200 $\mu \mathrm{m}, 250 \mu \mathrm{m}$ or $300 \mu \mathrm{m}$, and a $5 \mathrm{~mm}$ long cantilever for the measurements of a $490 \mu \mathrm{m}$ throat diameter nozzle. The demanded measurement length of at least $5.6 \mathrm{~mm}$ of the $800 \mu \mathrm{m}$ throat diameter nozzle exceeds the length of the existing longest cantilever $(5 \mathrm{~mm})$. This problem is solved by gluing two $5 \mathrm{~mm}$ long cantilevers together and obtaining a $7.5 \mathrm{~mm}$ long cantilever (see figure 6).

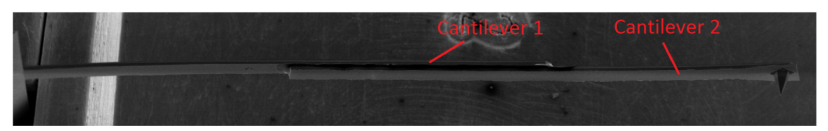

Figure 6. A $7.5 \mathrm{~mm}$ long cantilever obtained by gluing two 5 $\mathrm{mm}$ long cantilever

In the measurement, the nozzle was mounted on a Tform holder with the outlet facing the cantilever, and the holder was fixed on the sample stage. The parallelism between the central axis of the nozzle and the movement direction of the $\mathrm{X}$ piezo stage was assured by the T-form holder and better than $0.1^{\circ}$. Evenly distributed profile measurements indicate that the shape deviations of the nozzles are mainly rotationally symmetrical. Thus only one measured profile is presented for each nozzle in the following.

Because the maximum travel of the $\mathrm{X}$ piezo stage is only $800 \mu \mathrm{m}$, thus much smaller than the demanded measurement lengths, the stitching technique was employed to join several measurements at different positions into one profile. For example, in the measurements of nozzles with $800 \mu \mathrm{m}$ throat diameter, 11 measured profiles, each $772 \mu \mathrm{m}$ long and with 8192 data points, with starting position interval of $615 \mu \mathrm{m}$ along the axis, were stitched into a $6.9 \mathrm{~mm}$ long profile with about 73000 data points (see Figure 7). During the measurement, at first the cantilever was moved into the nozzle from the outlet by the $\mathrm{X}$ coarse stage without touching the surface till it reached the throat position. At this time the $\mathrm{X}$ piezo stage was at position 0 . Then the cantilever moved down and approached the inner surface and the $\mathrm{X}$ piezo stage scanned $772 \mu \mathrm{m}$ along the axis of the nozzle to the direction of the outlet with a scan rate of $20 \mu \mathrm{m} / \mathrm{s}$ and a constant probing force of $2 \mu \mathrm{N}$. At the end of the profile the cantilever was lifted up $5 \mu \mathrm{m}$ and the $\mathrm{X}$ piezo stage moved back to position 0 with a faster rate of $1 \mathrm{~mm} / \mathrm{s}$ to save measurement time and decrease the influence of thermal drift. Then the X coarse stage moved $615 \mu \mathrm{m}$ to the opposite direction of the outlet. The cantilever approached again and the second profile was measured. The procedure was repeated till all the 11 profiles (P1 to P11) were completed. 


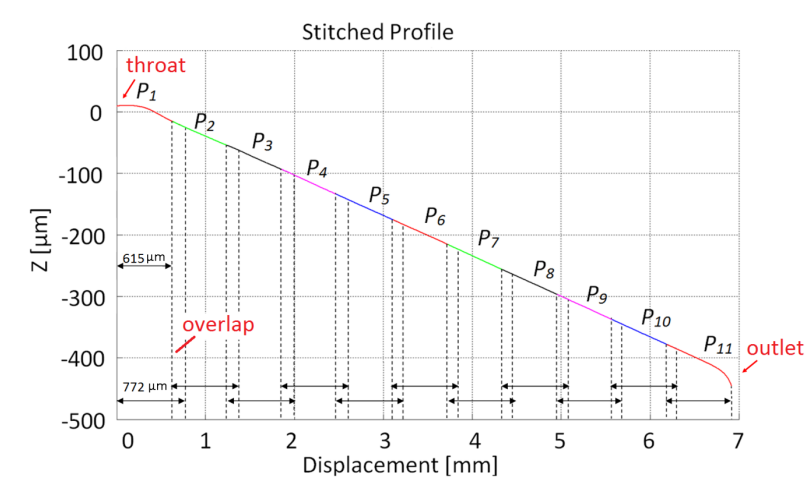

Figure 7. A $6.9 \mathrm{~mm}$ long profile was stitched using 11 measurements

Since a slope existed in the profiles and it would bring errors to the stitching results for higher values had more weights in stitching, the slope should be removed before stitching. The mean value of the slopes of all 11 profiles was calculated and removed from these profiles. Then the stitching was performed based on cross correlation. At last the removed slope was added back to the stitched profile.

The measured profiles of four nozzles with throat diameters of $800 \mu \mathrm{m}$ and $4^{\circ}$ diffuser opening angle are drawn in figure $8(\mathrm{a})$. For better comparison, the profile deviations from the ideal nozzle contour are presented. The axial position $x$ and profile height $z$ are normalized by the nozzle throat diameter $d$. The starting positions of the individual measurements vary slightly because the throat has no clearly defined starting point. The profiles of the four nozzles fluctuate obviously, which demonstrates that the manufacturing accuracy varied. The nozzle 0451 and 0294 were fabricated by turning. As mentioned above, their surfaces have obviously smaller roughness than the nozzle 0404 and 0497 which were produced by EDM. Except the roughness, the throat contours of nozzle 0404 and 0451 are quite similar. For the nozzle 0497, the profile next to the throat area, between the normalized axial position 1 and 2, has a crest (position $\mathrm{A}$ in the figure). The measured diffuser opening angle of the nozzle 0294 is about $7^{\circ}$, much steeper than the defined angle of $4^{\circ}$. Comparing the profile characters and the mass flow rate experiment results of the four nozzles, the impact of the above factors (surface roughness, exit cone opening angle deviation and crest structures next to the throat area) on the mass flow rate, can be investigated.

In figure $8(\mathrm{~b})$ are the corresponding gas mass flow measurement results of the four nozzles. The critical pressure ratio of the nozzle 0294 is higher than the other three nozzles but no "low unchoking" occurred with it. A strong downturn of the mass flow rate happened with the nozzle 0497 . The gas flow behaviour of the nozzle 0404 and 0451 are relatively similar although the surface roughness of the two nozzles is different. For both of the nozzles, "low unchoking" happened at a similar pressure ratio and the high unchoking curves at position $\mathrm{B}$ are very close to each other. It indicates that the surface roughness in this magnitude has no strong influence on the critical pressure ratio as well as the "low unchoking" behaviour.

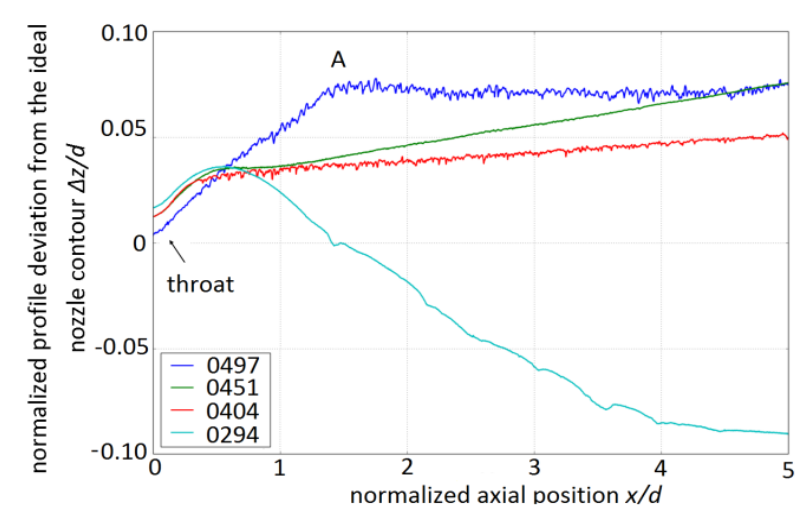

(a)

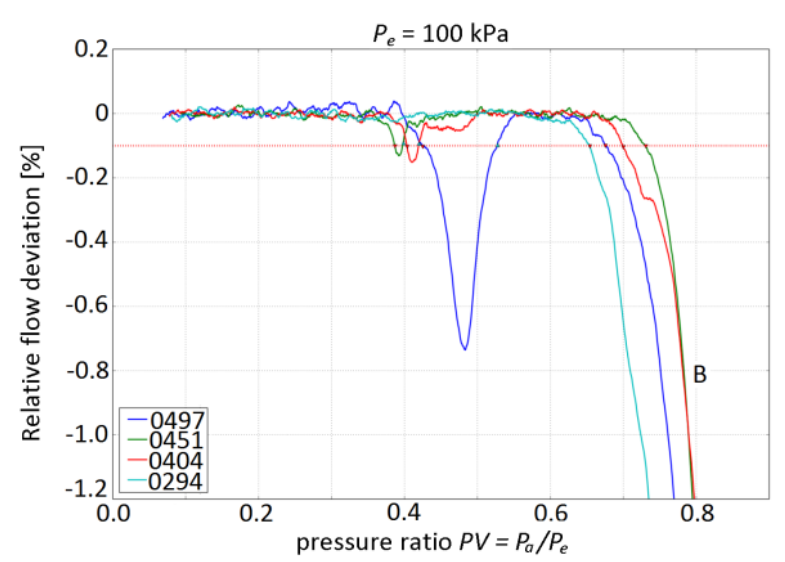

(b)

Figure 8. (a) The measured profiles of four nozzles with throat diameters of $800 \mu \mathrm{m}$ and $4^{\circ}$ diffuser opening angle; (b) the corresponding gas mass flow measurement results of the four nozzles.

To further prove the influence of the crest structures next to the throat area on the flow rate behaviour, two nozzles with quite similar contours except the topography next to the throat area were investigated. In figure 9(a) are the measured profiles of the two nozzles. In this figure the axial positions are normalized but the profile height not. Nozzle 1 had the crest structure next to the throat area while nozzle 2 had no such a structure in this area. In figure 9(b) the corresponding mass flow rate test results of the two nozzles are shown. The "low unchoking" happened to the nozzle 1 , but didn't occur to the nozzle 2 , and the critical pressure ratio of nozzle 2 was lower than that of nozzle 1 . The experimental results confirm the strong impact of the inner surface form of the nozzle on the "low unchoking" behaviour.

The measurements of the Profilscanner showed that the inner surface and shape of some micro nozzles deviated from the design. This leads to errors and unexpected behaviours in their mass flow properties. 


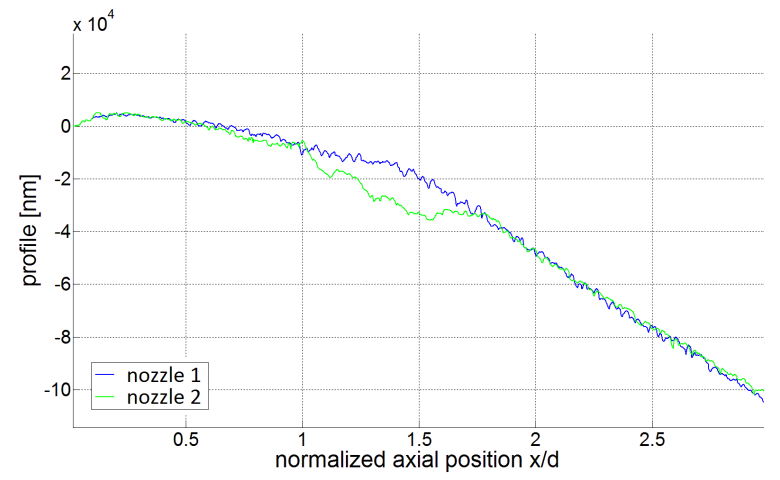

(a)

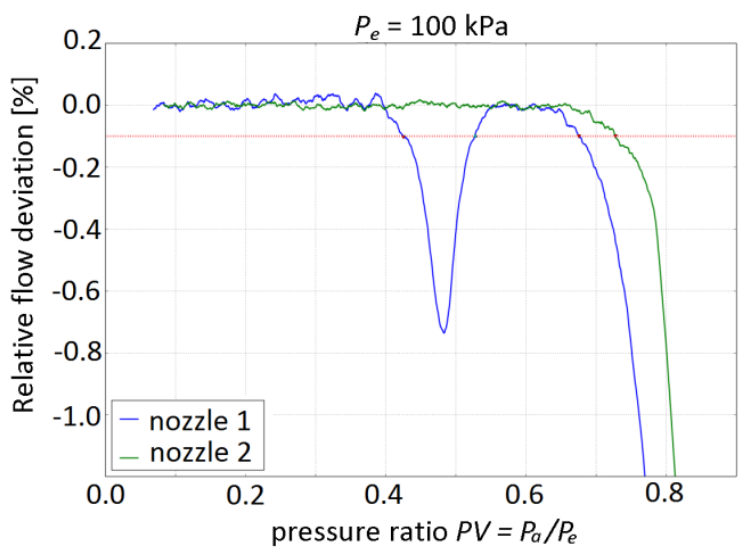

(b)

Figure 9. (a) The measured profiles of two nozzles with similar contours except at the position next to the throat; (b) the corresponding mass flow test results

\section{Conclusions}

A Profilscanner with the capability to traceably investigate the inner surface of small holes with the diameter down to $40 \mu \mathrm{m}$ has been developed and presented in the paper. For the first time, the inner surface of sonic nozzles with diameters in the micrometre range has been successfully characterized with the Profilscanner. Hereby, in some nozzles, manufacturing defects were detected which explained the deviation of the mass flow rate experiment results. This also confirms the assumption that the shape and the topography of the inner surfaces have a strong influence on the flow rate properties of the nozzles. It is expected that the calibration uncertainty and the quality control of such nozzles with diameters in the micrometre range can be improved by the measurement results of the Profilscanner.

\section{Acknowledgments}

The authors are grateful to the valuable assistance of Lena Jung-Albrecht, Hildegard Fischer, Susanne Schmidt, Radovan Popadic, Jürgen Kirchhoff and Thomas Ahbe during the instrument construction, roughness evaluation and cantilever imaging. Research was financial ly supported in part by the MNPQ programme, VII A5-07/07.

\section{References}

1. T. Masuzawa, Y. Hamasaki, and M. Fujino, CIRP Annuals-Manufacturing Technology, 42(1), 589-592 (1993)

2. T. Masuzawa, B. J. Kim, C. Bergaud and M. Fujino, CIRP Annals-Manufacturing Technoogy., 46(1), 437-440 (1997)

3. B. J. Kim, T. Masuzawa and T. Bourouina, Meas. S ci. Technol., 10, 697-705 (1999)

4. A. Weckenmann, G. Peggs and J. Hoffmann, Meas. Sci. Technol., 17, 504-9 (2006)

5. B. Muralikrishnan, J. A. Stone, and J. R. Stoup, Precision Eng., 30(2), 154-164 (2006)

6. H. Schwenke, F. Wäldele, C. Weiskirch and H. Kunzmann, CIRP Annals-Manufacturing Technology, 50(1), 361-364 (2001)

7. H. Murakami, A. Katsuki, H. Onikura, T. Sajima, N. Kawagoishi and E. Kondo, J. of Advanced Mechanical Design, Systems, and Manufacturing, 4(5), 995-1004 (2010)

8. J. Cui, L. Li and J. Tan, Meas. Sci. Technol., 23(8), 085105 (2012)

9. J. Cui, L. Li, J. Li and J. Tan, Sensors and Actuators A: Physical, 190, 13-18 (2013)

10. Y. Shimizu, B. $\mathrm{Xu}$ and W. Gao, Int. J. Nanomanufacturing, 8, 87-105 (2012)

11. F. G. Balzer, T. Hausotte, N. Dorozhovets, E. Manske and G. Jäger, Meas. Sci. Technol., 22(9), 094018 (2011)

12. K. Kiekens, F. Welkenhuyzen, Y. Tan, Ph. Bleys, A. Voet, J-P Kruth and W. Dewulf, Meas. Sci. Technol, 22, 115502 (2011)

13. M. Xu, J. Kirchhoff and U. Brand, Surf. Topogr. Metrol. Prop., 2(2), 024002 (2014)

14. ISO 9300:2005: Measurement of gas flow by means of critical flow Venturi nozzles.

15. I. Behrens, L. Doering and E. Peiner, J. Micromech. Microeng., 13, 171-7 (2003)

16. E. Peiner, M. Balke, L. Doering, U. Brand, H. Bartu ch and S. Völlmeke, Proc. Mikrosystemtechnik Kon gress, 369-74 (2007)

17. E. Peiner, M. Balke and L. Doering, IEEE Sensors J ournal, 8, 1960-7 (2008)

18. E. Peiner, M. Balke, L. Doering and U. Brand, Meas . Sci. Technol., 19, 064001 (2008)

19. E. Peiner and L. Doering, IEEE Sens. J., 13(2), 7018 (2013)

20. B. Mickan, R. Kramer and C. Li, 8th ISFFM, Colorado Springs, Colorado, USA, June $20-22$ (2012) 